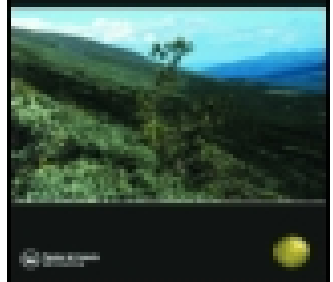

ISSN: 0374-6607 (Print) (Online) Journal homepage: http://www.tandfonline.com/loi/tped18

\title{
A New Leycesteria
}

\section{W. W. Smith}

To cite this article: W. W. Smith (1912) A New Leycesteria, Transactions of the Botanical Society of Edinburgh, 24:1-4, 173-175, DOI: 10.1080/03746601209468956

To link to this article: http://dx.doi.org/10.1080/03746601209468956

Published online: 29 Nov 2010.

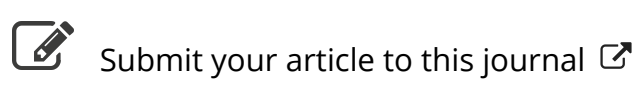

Џlll Article views: 2

Q View related articles 5 
the Corrie was a large patch of snow, and in its immediate neighbourhood vegetation was only just commencing. Large quantities of Pseud-athyrium alpestre were seen, and though most of it was much disfigured through being eaten down by the deer, a few interesting sub-varieties were found, which it is hoped will remain permanent under cultivation.

The heat of the sun may be judged by the fact that two of the members of the party bathed twice during the day in Loch Cander. Two other members who did not accompany the party to Glen Callater, found Gentiana campestris in the Glen near the hotel.

On July 15th, the only member of the party remaining spent the day among the ferns growing on the burn sides in the valley to the north of the hotel. Here he found a dwarf erect-growing variety of Lastrea montana with short pinnæ, similar plants of which have already been found on two previous occasions in Westmoreland, and which is known to fern growers by the name of angustata. Several large clumps of it were seen on the side of a burn within a space of about fifty yards. This variety keeps perfectly permanent in cultivation, and is a most interesting sport from the normal type of the ferm.

Though nothing very wonderful in regard to plants was found, the meeting will always remain memorable to the members present owing to the wonderful weather experienced, for although they have frequently had their pleasure spoilt and their botanising interfered with by rain and mist, it was quite a new experience, to most of them at all events, to have their work on the hills rendered almost unbearable owing to the extreme heat.

\section{A New Leycesteria. By W. W. Smith.}

Leycesteria Belliana, W. W. Smith, sp. nov.

Species Leycesteria sinensis Hems. valde affinis sed cum specimine unico ejus speciei (Henry 9692 c) in Herbario Kewensi non quadrat. Ad hoc confer Hook. Icones Plantarum, tab. 2633. Foliis stipulatis, petiolis brevissimis dense strigosis, inflorescentia brevispicata, calycis limbo 
5-partito, stylo piloso ab ea specie satis distinguenda. A Leycesteria stipulata (Hook. f. \& T.) Fritsch, habitu gracili, caule fistuloso glabrescente, stylo piloso inter alia differt.

Frutex ramosus paucipedalis, ramulis \pm albo-pilosulis, mox glabrescentibus, teretibus, fistulosis ad nodos septatis. Folia opposita breviter petiolata, membranacea, lanceolata, $5-10 \mathrm{~cm}$. longa, $1 \cdot 25-4 \mathrm{~cm}$. lata, acute caudato-acuminata, basi rotundata vel aliquantulum cordata, suprà subglabra vel parce strigillosa, subtus glauca, præcipue in venis \pm rufescenti-strigillosa, margine obscure sinuata, rarius ciliolata, venis primariis 5-7 paribus; petiolus $1-3 \mathrm{~mm}$. longus, dense strigosus; stipulæ parvæ ad $5 \mathrm{~mm}$. longæ, ad $3 \mathrm{~mm}$. latæ, rotundato-ovatæ, sæpe perpusillæ. Floris luteo-albidi $2 \mathrm{~cm}$. longi, sessilis, in spicas 2 -vel 4 -florigeras brevissimas ramulos laterales terminantes per paria dispositi pedunculis brevissimis; bracteæ bracteolæque plures foliaceæ rotundato-ovatæ vel lanceolatæ, floribus breviores. Calycis dense glanduloso-pilosi tubus $5 \mathrm{~mm}$. longus fusiformis; limbus fere ad imum in lobos quinque æquales, rarius subæquales, lineari - lanceolatos, acutos, $5 \mathrm{~mm}$. longos, persistentes divisus. Corolle $15 \mathrm{~mm}$. longa, subæqualiter 5-loba, hypocrateriformis, fere recta, extus glanduloso-pilosa, intus glabra, nisi infra mediam partem quā filamenta cum tubo conjuncta, lobis rotundatis $3-4 \mathrm{~mm}$. longis. Stamina brevissime exserta summo triente glabro parte cohærente villosula. Ovarium 5-loculare multiovulatum stylo stamina paulo superante filiformi, summo triente glabro, reliqua parte dense glanduloso-pilosa, stigmate capitato. Fructus immaturus angularis albidis glanduligeris capillis dense vestitus; semina vix matura $.75 \mathrm{~mm}$. longa, irregulariter ovoidea, subcompressa, minute punctulata, albida, nitentia.

Sikkim:-in monte Tonglo, 10,000 ped, alt., prope fines nepalenses 154 Herb. Sikkimense T. Anderson coll. anno 1862 ; inter montes pluviosos prope hospitium Karponang dictum, ad 9-10,000 ped. alt. 2996 Smithi. Typi in herbariis Kewensi et Edinburgensi et Calcuttensi conservati.

This species is rare in the Sikkim hills, and ranges higher than Leycesteria stipulata. The specific name is in honour of Mr A. C. Bell, I.C.S., British Resident in Sikkim, who has made botanical collections in Chumbi and elsewhere. It 

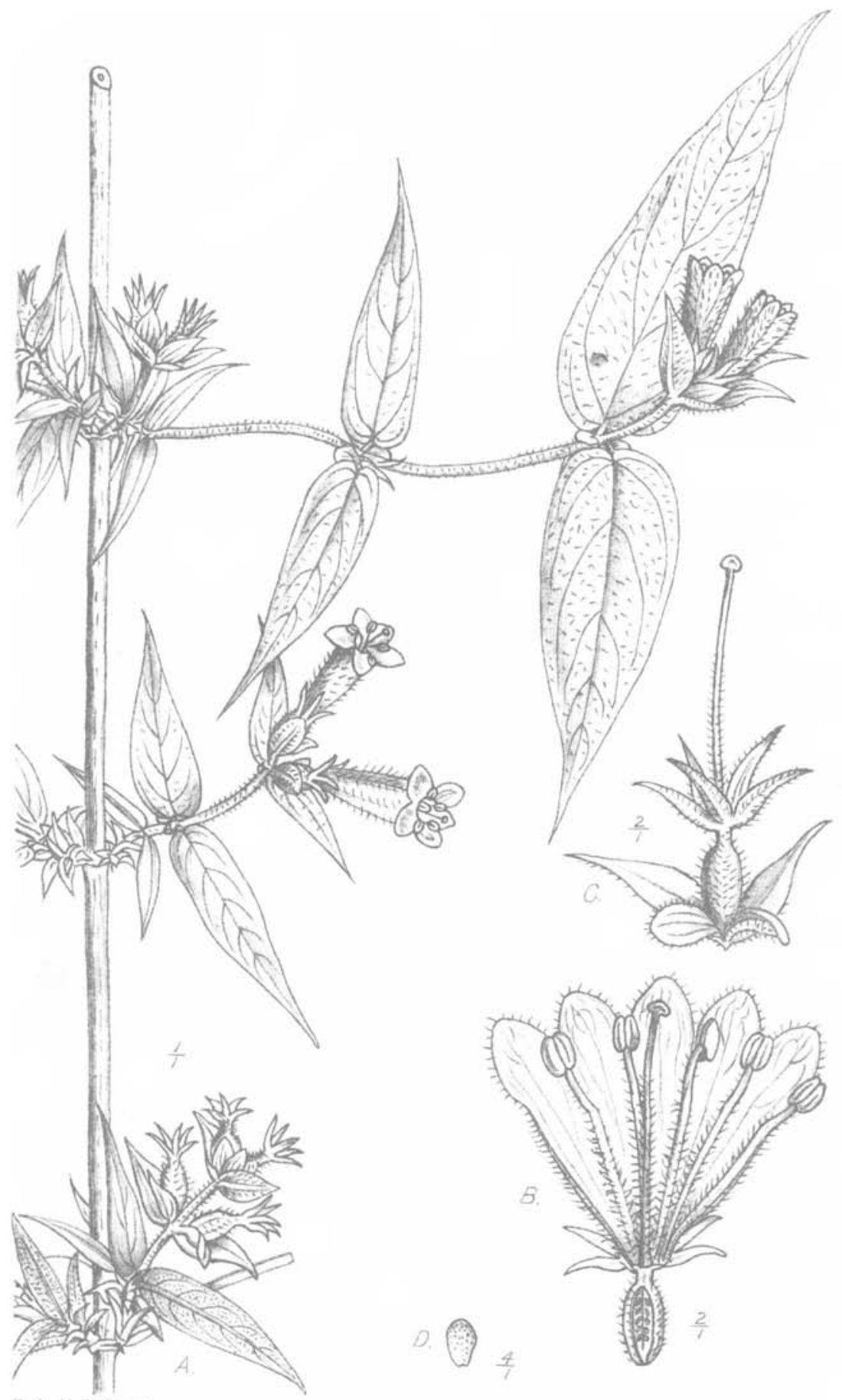

Del. K P Dass.

LEYCESTERIA BELLIANA, WW Smith 
ought to grow as well as Leycesteria formosa in European gardens, but, so far, seeds have not been available. I am indebted to $\mathrm{Mr}$ W. G. Craib, of Kew Herbarium, for kindly comparing the plant with Henry's Yunnan specimen.

\section{Borthwickia, a New Genus of Capparidacee. By W. W. Smith.}

Borthwickia genus novum.

Apud Capparidoideas-Capparideas (Pax); maturo fructu adhuc deficiente locus dubius, interim post. Ritchieam $R$. $B r$. positum est sed etsi frutex, ex affinitate Polanisice esse videtur. Frutex foliis oppositis trifoliatis; petala sex æqualia in latissimum unguem et laminam minorem discreta ; stamina in androphori brevis crassi apice inserta; fructus longe stipitatus 4-6 locularis. Species unica burmanica. Generis nomen honori cl. Alberti Borthwickii scientiæ doctoris et viri re botanica eruditi.

Borthwickia trifoliata, W. W. Smith, sp. nov.

Planta inermis, altitudine ignota, superior pars in scheda $40 \mathrm{~cm}$. longa. Caulis angulatus, striatus, subcompressus præsertim apud nodos ad $10 \mathrm{~cm}$. remotos, parce minute puberulus, mox glabrescens, racemo terminatus. Folia opposita, rarius subopposita, usque ad $8 \mathrm{~cm}$. petiolata, foliolis tribus lanceolatis acuminatis, basi cuneatis, 7-15 $\mathrm{cm}$. longis, $2-6 \mathrm{~cm}$. latis, $\mathbf{1 - 2} \mathrm{mm}$. petiolulatis, supra glabris, infra in nervis (6-9 paribus) nervulisque rufo-pilosulis. F'lores in terminalem racemum densum, in fructu ad $10 \mathrm{~cm}$. auctum, compositi. Bractece $1 \mathrm{~cm}$. longæ filiformes pilosulæ. Pedicelli $5 \mathrm{~mm}$. longi, rufo-pilosuli, in fructu ad $15 \mathrm{~mm}$. aucti. Sepala duo; neque in aperto flore neque in gemma aliorum interiorum sepalium vestigia vidi; $13-14 \mathrm{~mm}$. longa, 5-6 mm. lata, oblonga, obtusa, valvata, in aperto flore ad basim discreta, serius decidua. Petala sex, æqualia, 1-seriata (ideoque duo ut interiora sepala non habenda), valvata, divisa in unguem loriformem, $5 \mathrm{~mm}$. longum, $1.5 \mathrm{~mm}$. latum, villosulum, et in laminam, $3 \mathrm{~mm}$. longam, $1.5 \mathrm{~mm}$. latam, ovatam, glabram, obtusam, minute dentatam, 6-8 nervatam. Stamina in apice androphori $4-5 \mathrm{~mm}$. TRANS. BOT, SOC, FDIN, VOL, XXIV. 